\title{
AS CARACTERÍSTICAS DA GEOGRAFIA DA SAÚDE NO BRASIL: UMA ANÁLISE BIBLIOMÉTRICA DA PRODUÇÃO CIENTÍFICA NO PERÍODO DE 2012-2019
}

\section{The characteristics of health geography in Brazil: a bibliometric analysis of scientific production in the period 2012-2019}

\author{
Pollyana Furtado Machado Anute \\ Aluna do Programa de Mestrado em Geografia \\ Universidade Federal do Acre - UFAC \\ pollyana.anute@ifac.edu.br \\ lago Sales de Paula \\ Aluno do Programa de Mestrado em Geografia \\ Universidade Federal do Acre - UFAC \\ lago.ufac@gmail.com
}

\author{
Cleilton Sampaio de Farias \\ Doutor em Ensino de Biociências e Saúde - FIOCRUZ, \\ Professor do Programa de Mestrado em Geografia \\ Universidade Federal do Acre - UFAC \\ cleilton.farias@ifac.edu.br
}

\begin{abstract}
RESUMO: A geografia da saúde é uma área de estudos que tem como objetivo investigar, interpretar e explicar o modo como os fatores sociais e as desigualdades socioeconômicas afetam as questões de saúde de uma determinada população. Visando compreender melhor as áreas de estudo dessa disciplina na atualidade, realizamos uma revisão bibliométrica dos artigos publicados na revista Hygeia, visto que a mesma é uma referência em Geografia da Saúde. Para isso, analisamos cada um dos artigos publicados no período de 2012 a 2019, identificando as instituições, os estados e as regiões que mais publicaram na revista, o gênero dos autores, suas titulações, as vertentes e abordagens geográficas e as doenças mais recorrentes nas pesquisas. Todos os dados foram tabulados no Excel, cruzados e transformados em gráficos para melhor facilitar a discussão. Verificamos que a região que mais publica na revista é a Sudeste, que o número de homens e de mulheres que publicam na revista estão muito próximos, que grade parte dos autores possui doutorado, que os artigos que possuem uma abordagem de análise espacial sempre direcionam seus estudos para a vertente da atenção médica, e os artigos que possuem uma abordagem ecológica analisam sempre os fenômenos a partir da abordagem nosogeográfica. Por fim, as doenças mais recorrentes nos artigos são a dengue, a Leishmaniose visceral e a hanseníase.
\end{abstract}

Palavras-chave: Geografia da Saúde; Revista Hygeia; Levantamento Bibliométrico.

ABSTRACT: Health geography is an area of study that aims to investigate, interpret and explain how social factors and socioeconomic inequalities affect the health issues of a given population. In order to better understand the areas of study of this discipline nowadays, we carried out a bibliometric review of the articles published in the journal Hygeia, since it is a reference in Health Geography. For that, we analyzed each of the articles published in the period from 2012 to 2019, identifying the institutions, states and regions that most published in the journal, the gender of the authors, their titles, the 
geographical aspects and approaches and the most recurrent diseases in research. All data were tabulated in Excel, crossed and transformed into graphs to better facilitate the discussion. We found that the region that publishes the most in the journal is the Southeast, that the number of men and women who publish in the journal are very close, that a large part of the authors have a doctorate, that articles that have a spatial analysis approach always direct their studies for medical care, and articles that have an ecological approach always analyze the phenomena from the nosogeographic approach. Finally, the most recurrent diseases in the articles are dengue, visceral Leishmaniasis and leprosy.

Keywords: Health Geography; Hygeia magazine; Bibliometric survey.

\section{INTRODUÇÃO}

A Geografia da Saúde, ou Geografia Médica (dependendo da escola), é uma área de estudos da geografia que tem como objetivo investigar, interpretar e explicar o modo como os fatores sociais e as desigualdades socioeconômicas afetam as questões de saúde de uma determinada população, estudando a dimensão geográfica relativa a tais resultados de modo a direcionar da melhor maneira possível as políticas públicas e os investimentos financeiros necessários à promoção de um sistema de saúde eficaz (SANTANA, 2014).

De acordo com Armstrong (1983), os primeiros dados de estudos relacionados ao que hoje denominamos de Geografia da Saúde surgiram através dos primeiros registros sobre as variações de saúde de diversas populações que residiam em diferentes lugares. Tais registros eram feitos por médicos viajantes que anotavam os detalhes das cidades pelas quais passavam e as características de suas populações, fazendo uma correlação entre tais fatores e o modo como os mesmos influenciavam no surgimento ou na disseminação de certas enfermidades. Podemos citar o tratado de Hipócrates ("Dos Ares, das Águas e dos Lugares", 480 a.C.) como um dos primeiros escritos a abordar essa relação entre a saúde e os "lugares". Era essencial conhecer não apenas a doença em si, mas também o entorno no qual a mesma ocorria.

\begin{abstract}
[...] o estudo do enfermo é inseparável do seu ambiente, do biótopo onde se desenvolvem os fenômenos de ecologia associada com a comunidade a que ele pertence. Quando se estuda uma doença, principalmente metaxênica, sob o ângulo da geografia médica, devemos considerar, ao lado do agente etiológico, do vector, do reservatório, do hospedeiro intermediário e do Homem suscetível, os fatores geográficos representados pelos fatores físicos (clima, relevo, solos, hidrografia, etc.), fatores humanos ou sociais (distribuição e densidade da população, padrão de vida, costume religioso superstições, meios de comunicação) e os fatores biológicos (vidas vegetal e animal, parasitismo humano e animal, doenças predominantes, grupo sanguíneo da população, etc.). (BARUZZI; SIQUEIRA JÚNIOR, 1972, p. 01 apud SANTOS, 2010, p. 43).
\end{abstract}

A partir do século XIX, pesquisas e trabalhos realizados por médicos e profissionais da área da saúde têm sido de grande importância para que possamos compreender o contexto das enfermidades e da saúde de um modo geral.

REVISTA GEONORTE, V.12, N.40, p.41-58, 2021.

DOI: 10.21170/geonorte.2021.V.12.N.40.41.58

(ISSN 2237 - 1419) 


\begin{abstract}
Desde meados do século XIX até ao presente que se pode demonstrar, através de trabalhos de investigação fundamentalmente desenvolvidos por médicos, a utilidade da Geografia no estudo dos problemas da saúde e da doença e de acesso aos cuidados de saúde. Por exemplo, em 1850, John Snow usou o mapa dos casos de cólera em Londres para identificar o poço contaminado como fonte da epidemia. Mais tarde, Edward Jarvis (1866) verificou uma tendência consistente de diminuição da utilização per capita dos hospitais mentais com a distância. No livro Health Care delivery: spatial perspectives (1974), Gary Shannon e Alan Dever apresentam os resultados de um estudo que refere o efeito da distância (da residência ao local de prestação de cuidados), na frequência de consultas, práticas de referenciação, demora média no internamento e em outros aspectos dos cuidados de saúde. São, ainda, apresentadas técnicas de descrição e interpretação dos dados, como a teoria dos lugares centrais, os modelos da distância - diminuição da utilização e modelos gravitacionais. (SANTANA, 2014, p. 14.)
\end{abstract}

Durante muito tempo utilizou-se o termo Geografia Médica para designar a análise de fenômenos mais relacionados às enfermidades que assolavam determinada população a partir da análise exclusiva do entorno físico (clima, temperatura, relevo, vegetação, entre outros), pois os médicos responsáveis por tais investigações desconheciam os agentes etiológicos e microbianos das doenças. Ou seja, a relação entre a saúde e o lugar era muito determinista, predominando, maiormente as relações de causa e efeito com o ambiente físico.

Com o avanço dos estudos sobre a microbiologia, várias transformações ocorreram no campo da medicina. Os termos saúde e doença passaram a receber um novo olhar por parte dos médicos e demais profissionais. O corpo humano passa a ser a prioridade na formação médica e não mais o meio ambiente, o que faz com que a geografia médica caia em desuso e seja excluída dos currículos disciplinares.

Todavia, no final do século XIX, surgiram novas tentativas de relacionar a Geografia Médica com as epidemiologias. O ponto alto dessa relação foi o importante trabalho elaborado por John Snow (1854), sobre um surto de cólera que acometeu a cidade de Londres em 1848. Utilizando a estatística descritiva e um mapeamento detalhado, Snow localizou as áreas onde haviam os maiores índices de cólera em diferentes áreas servidas pelas companhias de água, concluindo que a presença de um foco infeccioso se encontrava na região de Broad Street. Com isso ele demonstrou que a água era o fator de transmissão do Cólera (PEITER, 2005).

Com o advento do positivismo, a geografia médica entra novamente em declínio, pois os seus métodos não eram considerados "científicos", visto que não eram analisados à luz dessa nova corrente filosófica. Muitos trabalhos eram vistos com desconfiança por terem um caráter muito determinista (NOSSA, 2001). No entanto, com o início da Segunda Guerra Mundial, a geografia médica ganha um novo impulso, pois fez-se necessário identificar as doenças encontradas nos campos de batalha.

Com o surgimento das teorias neopositivistas pós-guerra, os modelos ecológicos se tornam uma prioridade, e a geografia médica passa por uma nova etapa de 
AS CARACTERÍSTICAS DA GEOGRAFIA DA SAÚDE NO BRASIL: UMA ANÁLISE BIBLIOMÉTRICA DA PRODUÇÃO CIENTÍFICA NO PERÍODO DE 2012-2019

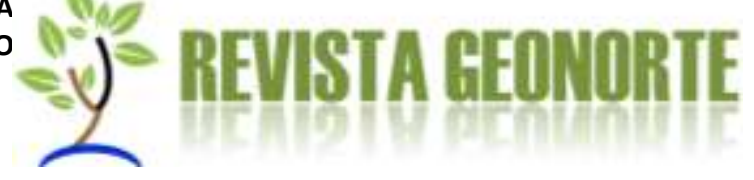

reconstrução de seus métodos e modelos, mais adequados às exigências quantitativas e matemáticas. Tais métodos focalizam agora na investigação e transmissão de patologias infecciosas tendo o SIG (Sistema de Informação Geográfica) como principal aliado tanto de médicos quanto de geógrafos que se debruçavam sobre tais estudos.

Com o passar do tempo, o termo geografia médica começa a ser questionado, pois entendia-se que tal denominação era muito determinista e não representava o leque de abordagens que podem ser feitas a partir desses estudos. Defende-se então que tal disciplina passe a ser alcunhado de Geografia da Saúde, pois compreende-se que tal nomenclatura aborda muito melhor os estudos e análises que vinham sendo realizados, como explica Peiter (2005, p. 05):

\begin{abstract}
A denominação da disciplina por várias décadas foi Geografia Médica, mas a ampliação do espectro de temas, questões e abordagens que esta foi desenvolvendo ao longo do tempo desembocou no pedido da Comissão de Geografia Médica da União Geográfica Internacional (UGI), em Moscou (1976), de se adotar uma nova denominação mais abrangente, substituindo Geografia Médica por Geografia da Saúde. A nova designação não vingou em todos os países, encontrando-se ainda hoje a denominação Geografia Médica em alguns países anglo-saxões.
\end{abstract}

De acordo com Santana (2014) o desenvolvimento da Geografia da Saúde se deu através das publicações realizadas nas revistas Social Science and Medicine e Health and Place, nas quais os geógrafos e demais profissionais que se debruçavam sobre tal área tiveram voz e espaço para exporem suas pesquisas. Os principais problemas tratados na época (e que ainda permanecem muito atuais, se compararmos com as publicações mais recentes) eram a distribuição e difusão de doenças, a localização dos equipamentos públicos, as determinantes do estado de saúde da população, os conflitos entre setores públicos e privados e o controle com as despesas relacionadas à saúde. Todos esses problemas revelam a necessidade de reflexões a respeito de um melhor planejamento dos serviços de saúde.

O Objeto da Geografia da Saúde e dos cuidados de saúde é relativo ao estudo geográfico da distribuição e acesso dos serviços de saúde e à avaliação das desigualdades em saúde. Têm vindo a ser privilegiados alguns temas como a organização, acesso e utilização dos cuidados de saúde e, mais recentemente, a otimização da localização dos equipamentos de saúde e planificação de serviços de saúde. (SANTANTA, 2014. p. 19, grifo da autora)

Desde os anos 1970 a Geografia da Saúde vem assumindo grande importância principalmente nas escolas anglo-saxônicas, francesa, alemã e russa. No Brasil, essa área de estudos crescendo cada dia mais. Tal evolução se deve ao fato de que a geografia da saúde permite a análise da sociedade de uma forma ampla, "[...] porque o campo da saúde oferece ao geógrafo a oportunidade de analisar a sociedade de forma real, debruçando-se sobre as desigualdades perante a morte, a doença e a saúde, articulando factos naturais e factos sociais [...]" (SANTANA, 2014, p. 04). 
É importante ressaltar que a geografia possui como um de seus principais desafios conciliar suas áreas física e humana de modo que uma baseie os estudos da outra. São poucas as disciplinas que conseguem esse feito de forma bem sucedida, e a geografia da saúde é uma delas, pois seu campo de estudo quase sempre analisa fatores do ambiente físico a fim de explicar fenômenos ocorridos em determinadas populações, como doenças, recursos médicos, qualidade de vida, entre outros. Além disso, seus estudos e investigações passam a ser amplamente utilizados por outras áreas, como saúde pública e planejamentos políticos e governamentais.

O caráter transdisciplinar atribui à Geografia da saúde, por um lado, atualidade no quadro da investigação científica e, por outro, dá-lhe qualidade para se tornar cada vez mais uma disciplina fundamental na Geografia atual e futura. De facto, a Geografia da Saúde constitui-se como uma área de sínteses proveitosas e aí reside grande parte da sua força e importância no atual contexto da Geografia, contrariando a tendência decorrente da crescente especialização, que constitui uma ameaça à integridade e ao futuro da Geografia, pelo risco de sua fragmentação (SANTANA, 2005, p. 13).

No Brasil, a Geografia da Saúde merece destaque por conseguir conciliar tão bem questões socioeconômicas e ambientais com a saúde da população. Christovam Barcellos, Helena Ribeiro, Lígia Barroso, Raul Guimarães e Samuel Lima são os principais nomes quando se fala nesse campo de estudos no Brasil, sendo eles os pioneiros nos estudos voltados a essa disciplina em nosso país.

No Brasil, desde meados do séc. $X X$ que vários investigadores desenvolvem trabalhos de pesquisa que têm como objetivo relacionar as condições socioeconómicas e ambientais com a saúde da população. Os primeiros trabalhos são desenvolvidos por médicos, com destaque para os de Samuel Pessoa (Ensaios Médico-Sociais, 1978). Nas últimas décadas do séc. XX salientam-se autores como Christovam Barcellos (Barcellos, 2008), Carlos Lacaz (Lacaz et al., 1972), Helena Ribeiro (1 ${ }^{a}$ tese de doutoramento em Geografia da Saúde (Ribeiro, 1988)), Lígia Barroso, Raul Guimarães e Samuel Lima. A Geografia da Saúde no Brasil assume cada vez maior importância e esse facto está associado aos eventos científicos que reúnem centenas de geógrafos periodicamente. O primeiro Simpósio Nacional de Geografia da Saúde/ Fórum Internacional de Geografia da Saúde aconteceu em 1993, em Presidente Prudente. Em 2013, realizou-se o VI Simpósio, em São Luis do Maranhão. (SANTANA, 2014, p. 19).

De acordo com Peiter (2005) as duas principais vertentes da geografia da saúde são a Nosogeografia e a Geografia da Atenção Médica. A primeira, tida como um ramo mais tradicional, procura identificar e analisar os padrões de distribuição espacial das doenças. Já a segunda vertente se dedica à distribuição e ao planejamento dos componentes infra estruturais e dos recursos humanos do Sistema de Atenção Médica.

Tratando-se agora de abordagens, podemos afirmar que a Geografia da Saúde se utiliza de dois principais modelos que visam compreender o processo de 
AS CARACTERÍSTICAS DA GEOGRAFIA DA SAÚDE NO BRASIL: UMA ANÁLISE BIBLIOMÉTRICA DA PRODUÇÃO CIENTÍFICA NO PERÍODO DE 2012-2019

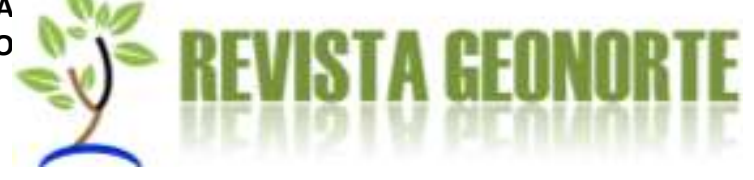

saúde/doença no espaço: a Abordagem Ecológica e a Abordagem de Análise Espacial (Peiter, 2005).

A Abordagem Ecológica é uma das mais recorrentes na Geografia da Saúde e possui seus modelos baseados no "meio geográfico" de Vidal de La Blache. Defende-se que as análises sobre as doenças não devem se basear apenas no ambiente físico, mas também nas relações sociais, originando o termo "complexos patogênicos" por Max Sorre (1933). O autor enfatiza a importância da paisagem, que é por onde circulam os agentes infecciosos. Essa abordagem se desenvolve principalmente nos Estados Unidos (1950), por Jacques May. "Em suma, a abordagem ecológica das doenças foi fundamental para o desenvolvimento da Geografia da Saúde a partir de meados do século $X X$, tendo na figura de $M$. Sorre seu maior expoente" (PEITER, 2005, p. 24).

Armstrong (1983) nos explica que a Abordagem de Análise Espacial procura aplicar métodos matemáticos com o intuito de esclarecer as relações socioespaciais entre a saúde e a distribuição espacial da população, buscando criar padrões que podem ter uma aplicação geral Defende-se a ideia de que os índices de morbimortalidade e saúde não se dão de maneira aleatória, mas seguem um determinado padrão que irá depender da sua população e das possíveis causas adjacentes, como os serviços de saúde e a qualidade do atendimento médico. Em síntese essa abordagem não foca sua análise sobre o ambiente físico, mas procura entender o modo como a distribuição dos serviços de saúde influencia na qualidade de vida de uma determinada população.

Feita essa breve conceituação teórica, uma questão surge: quais as principais características dessa área atualmente no Brasil? Há várias formas de responder a questão, entretanto, acreditamos que a análise de artigos publicados em uma revista especializada na temática pode ser um bom caminho.

Nesse sentido, objetivamos expor e discutir os resultados obtidos a partir de um levantamento bibliométrico realizado na revista Hygeia, nos artigos publicados, durante os anos de 2012 a 2019. A revista Hygeia se configura na atualidade como um dos principais meios para a divulgação de trabalhos científicos voltados para a área da Geografia Médica e da Saúde. Pertencente ao Programa de Pós-graduação em Saúde Ambiental e Saúde do Trabalhador da Universidade Federal de Uberlândia, está sob a chancela da Editora da Universidade Federal de Uberlândia (EDUFU). Iniciou suas publicações no ano de 2005 e possui periodicidade trimestral. Atualmente se encontra classificada como B1 para a Geografia.

Os levantamentos bibliométricos estão em voga no meio científico e acadêmico, pois os mesmos nos permitem analisar de maneira rápida e eficaz os principais meios de publicação cientifica da atualidade: os periódicos. Tal metodologia nos permite identificar quais as direções que os estudos e pesquisas estão tomando nas mais diversas áreas.

Escolhemos aplicar tal método tendo como base a revista Hygeia para melhor compreendermos o modo como a Geografia Médica e da Saúde se encontra nos dias atuais. Para isso extraímos dados como instituições, autores, formação 
AS CARACTERÍSTICAS DA GEOGRAFIA DA SAÚDE NO BRASIL: UMA ANÁLISE BIBLIOMÉTRICA DA PRODUÇÃO CIENTÍFICA NO PERÍODO DE 2012-2019

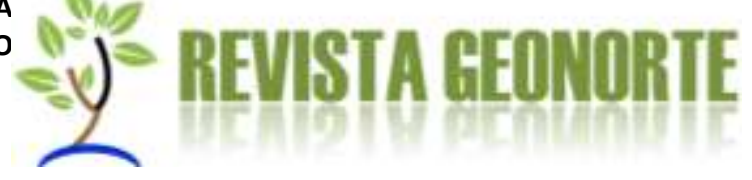

acadêmica, doenças, entre outros. Utilizamos como recorte temporal o período de 2012 a 2019, visto que o período anterior a esse já possui trabalhos e levantamentos sobre essa mesma revista.

\section{MATERIAIS E MÉTODOS}

De acordo com Maranhão (2014) os levantamentos bibliométricos estão em voga na atualidade, ganhando cada vez mais espaço no meio acadêmico e até governamental. Para o autor a bibliometria nada mais é que a contagem de atividades bibliográficas, que dá a possibilidade de estudo de termos específicos, como por exemplo, autores, instituições, número de citações, entre outros pontos que possam ser de interesse do pesquisador.

Segundo Araújo e Alvarenga (2011) a pesquisa bibliométrica consiste em realizar-se um levantamento de técnicas de investigação de informações, que posteriormente são apresentadas, permitindo aos pesquisadores a compreensão da evolução do método e tendências no campo de estudos pesquisado. Assim, a importância de pesquisas bibliométricas,

[...] pode ser notada ao analisar a seguinte lista de possibilidades de
aplicação, uma lista que não se destina a ser completa. Identifique
tendências e crescimento do conhecimento em diferentes disciplinas.
Estime a cobertura de revistas secundárias. Identifique os usuários das
diferentes disciplinas. Identifique autores e tendências em diferentes
disciplinas. Avalie a utilidade dos serviços de divulgação seletiva de
informaçães. Prever tendências de publicação. Identifique as revistas do
núcleo de cada disciplina. Formule políticas de compras que respeitem o
orçamento. Adapte as políticas de descarte de publicação. Estudar a
dispersão e obsolescência da literatura científica. Padrões de design para
padronização. Crie processos automáticos de indexação, classificação e
resumo. Preveja a produtividade de editores, autores individuais,
organizações, países etc. (SPINAK, 1996, p. 06).

Assim, na nossa pesquisa, os dados foram extraídos no acervo eletrônico da revista Hygeia (http://www.seer.ufu.br/index.php/hygeia/issue/archive) publicados no período de 2012 a 2019. Todas as informações foram tabuladas e organizadas em planilhas, tomando por base as seguintes informações: título, ano de publicação, instituição dos autores, unidade federativa dos autores, região dos autores, quantidade de autores, gênero dos autores, formação acadêmica dos autores, titulação dos autores, abordagem da Geografia da Saúde utilizada (Ecológica ou Análise Espacial), vertente da Geografia da Saúde utilizada (Nosogeografia ou Geografia da Atenção Médica) e as doenças pesquisadas. Após tabuladas e devidamente organizadas, todas as informações foram transformadas em gráficos, tabelas e nuvem de palavras para melhor facilitar a discussão e a compreensão dos dados.

Ao todo, foram analisados 241 artigos publicados durante o período de 2012 a 2019 . Escolhemos esse recorte temporal pelo fato de os anos anteriores já possuírem trabalhos dessa mesma modalidade. 
AS CARACTERÍSTICAS DA GEOGRAFIA DA SAÚDE NO BRASIL: UMA ANÁLISE BIBLIOMÉTRICA DA PRODUÇÃO CIENTÍFICA NO PERÍODO DE 2012-2019

\section{泣}

Inicialmente realizamos o levantamento sobre a quantidade de artigos que foram enviadas de cada região do Brasil para a publicação na revista. Para isso, fez-se um minucioso levantamento de todas as instituições que elaboraram tais trabalhos assim como os estados nos quais cada uma delas se encontra localizada.

Achamos importante também investigar o gênero dos autores que estão publicando na revista (feminino ou masculino), visando identificar se há predominância de um sobre o outro ou se há igualdade entre trabalhos publicados por homens e por mulheres nesse campo cientifico. Em seguida analisamos a titulação de cada um dos autores e coautores. Tendo em vista que nem todos os artigos detalhavam tal informação, recorremos à plataforma de pesquisa do currículo Lattes (http://buscatextual.cnpq.br/buscatextual/busca.do?metodo=apresentar) com base no ano de publicação do artigo para fazer esse levantamento de titulações.

Analisamos também a vertente geográfica de cada um dos artigos, buscando identificar qual delas predominava sobre as pesquisas, a vertente da Atenção Médica ou a vertente Nosogeográfica. Concomitantemente a tal levantamento, investigamos também as abordagens geográficas mais recorrentes nos trabalhos, sendo Abordagem de Análise Espacial ou Abordagem Ecológica, buscando com isso realizar um cruzamento de dados dessas vertentes e abordagens de modo a traçar um padrão.

Por fim, realizou-se um levantamento sobre as doenças que mais aparecem nos artigos, visando com isso identificar aquelas que estão sendo mais recorrentes e despertando maior interesse por parte dos pesquisadores.

\section{RESULTADOS E DISCUSSÕES}

Apresentaremos agora os resultados obtidos através da pesquisa realizada assim como as devidas discussões sobre os dados obtidos através desse levantamento bibliométrico realizado na Revista Hygeia.

Como podemos observar na figura 01, no ano de 2012 a revista teve apenas uma edição (dezembro), o que explica a pequena quantidade de artigos analisados em relação aos anos seguintes. A partir de 2013 a revista passou a publicar semestralmente. Nos anos de 2017 e 2018 a revista teve, excepcionalmente, 03 edições, o que explica o fato de tais anos aparecerem como os que mais possuem artigos analisados. 
AS CARACTERÍSTICAS DA GEOGRAFIA DA SAÚDE NO BRASIL: UMA ANÁLISE BIBLIOMÉTRICA DA PRODUÇÃO CIENTÍFICA NO PERÍODO DE 2012-2019

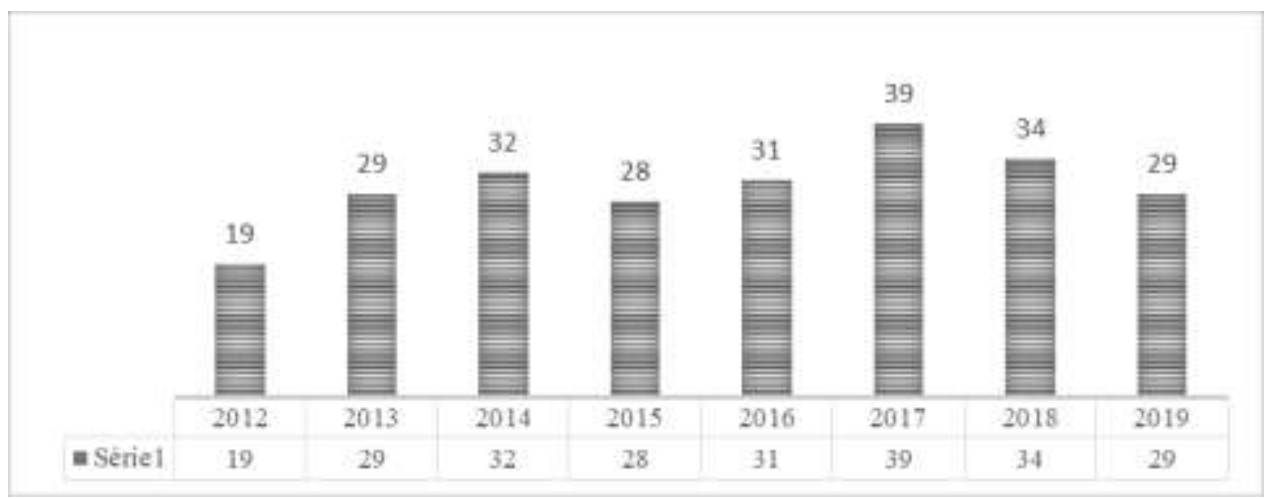

Figura 01. Número de artigos publicados por ano. Fonte: Elaborado pelos autores (2021).

A região que mais publicou na revista Hygeia durante esse período foi o Sudeste. Isso se deve ao fato de a referida revista pertencer à Universidade Federal de Uberlândia, localizada no estado de Minas gerais. Tal universidade possui um curso de graduação em geografia com enfoque na área de saúde. Além da Universidade Federal de Uberlândia, vários outros cursos superiores da região Sudeste possuem um enfoque muito grande na área de estudos e pesquisas em saúde (Figura 2).

Outra região que aparece com grande destaque nesse cenário é o Nordeste, e isso se deve ao fato de a geografia da saúde estar sendo implantada em algumas instituições de nível superior e nos cursos de pós-graduação. Podemos citar como exemplo a Universidade Federal do Rio Grande do Norte, que se destaca dentro da região como sendo a que mais publicou artigos na revista durante o período de 2012 a 2019. Além dela, vale citar também as parcerias feitas pela Fundação Oswaldo Cruz (FIOCRUZ) com diversas instituições da região.

Já a região que aparece de forma mais tímida é o Norte, e isso pode ser explicado pelo fato de nessa região os cursos que envolvem a disciplina de geografia da saúde serem muito recentes (Figura 02).

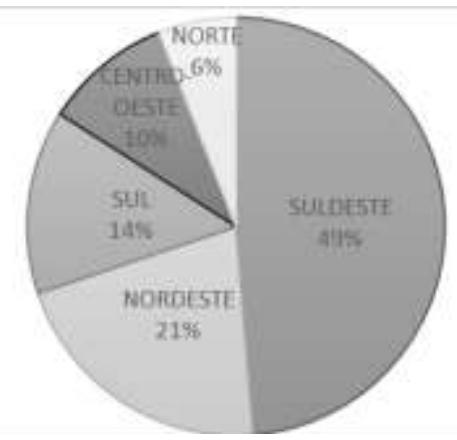

Figura 02. Percentual de artigos publicados na revista Hygeia por região. Fonte: Elaborado pelos autores (2021).

Além da universidade Federal de Uberlândia, a Universidade de São Paulo (USP), com a disciplina de Espaço e Saúde, tem despontado bastante na área de 
AS CARACTERÍSTICAS DA GEOGRAFIA DA SAÚDE NO BRASIL: UMA ANÁLISE BIBLIOMÉTRICA DA PRODUÇÃO CIENTÍFICA NO PERÍODO DE 2012-2019

especializações em saúde, sem falar da Fundação Oswaldo Cruz (FIOCRUZ), no Rio de Janeiro, referência nacional nos estudos e pesquisas nessa mesma área. Tudo isso explica o fato de a região sudeste estar na liderança do número de publicações na revista Hygeia, em especial os estados de Minas Gerais, Rio de Janeiro e São Paulo, como demonstra a figura 03.

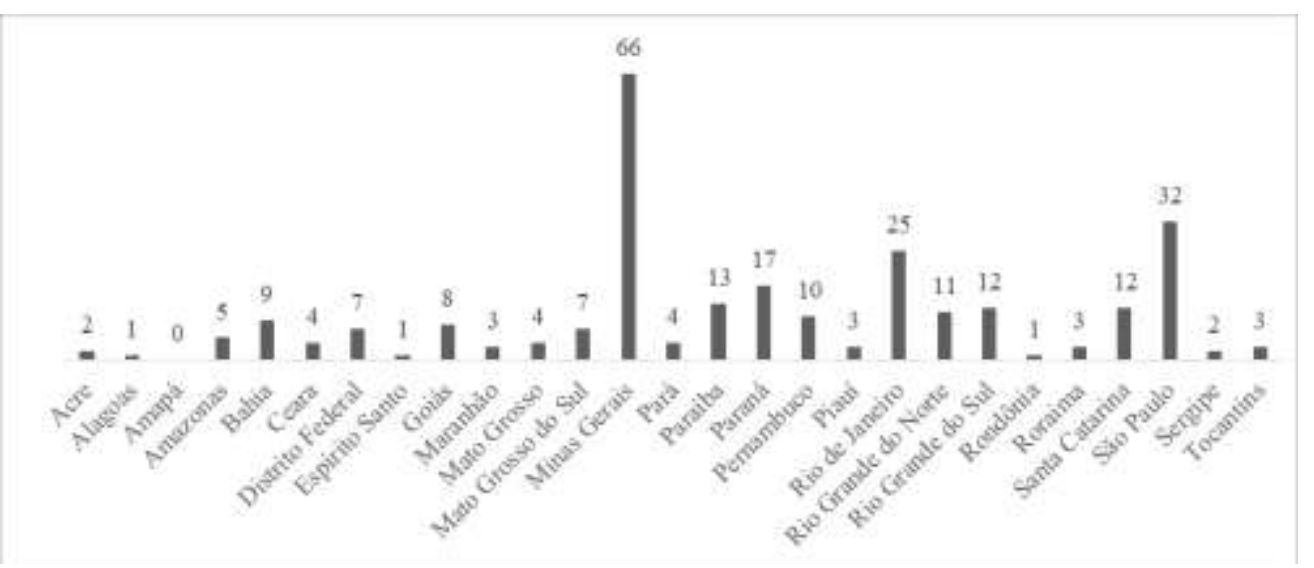

Figura 03. Quantidade de artigos publicados por estado. Fonte: Elaborado pelos autores (2021).

Como explicado anteriormente, os autores que mais publicam na revista Hygeia, fazem parte das universidades mostradas na figura 04. Além das já citadas anteriormente (UFU, USP e FIOCRUZ) merecem destaque também a Universidade Federal do Rio Grande do Norte, a Universidade Federal de Brasília e a Universidade Comunitária da Região de Chapecó. No gráfico abaixo, elencamos as principais universidades por estado, ou seja, aquela que dentro do seu estado foi a que mais apareceu nas publicações da revista. 
AS CARACTERÍSTICAS DA GEOGRAFIA DA SAÚDE NO BRASIL: UMA ANÁLISE BIBLIOMÉTRICA DA PRODUÇÃO CIENTÍFICA NO PERÍODO DE 2012-2019

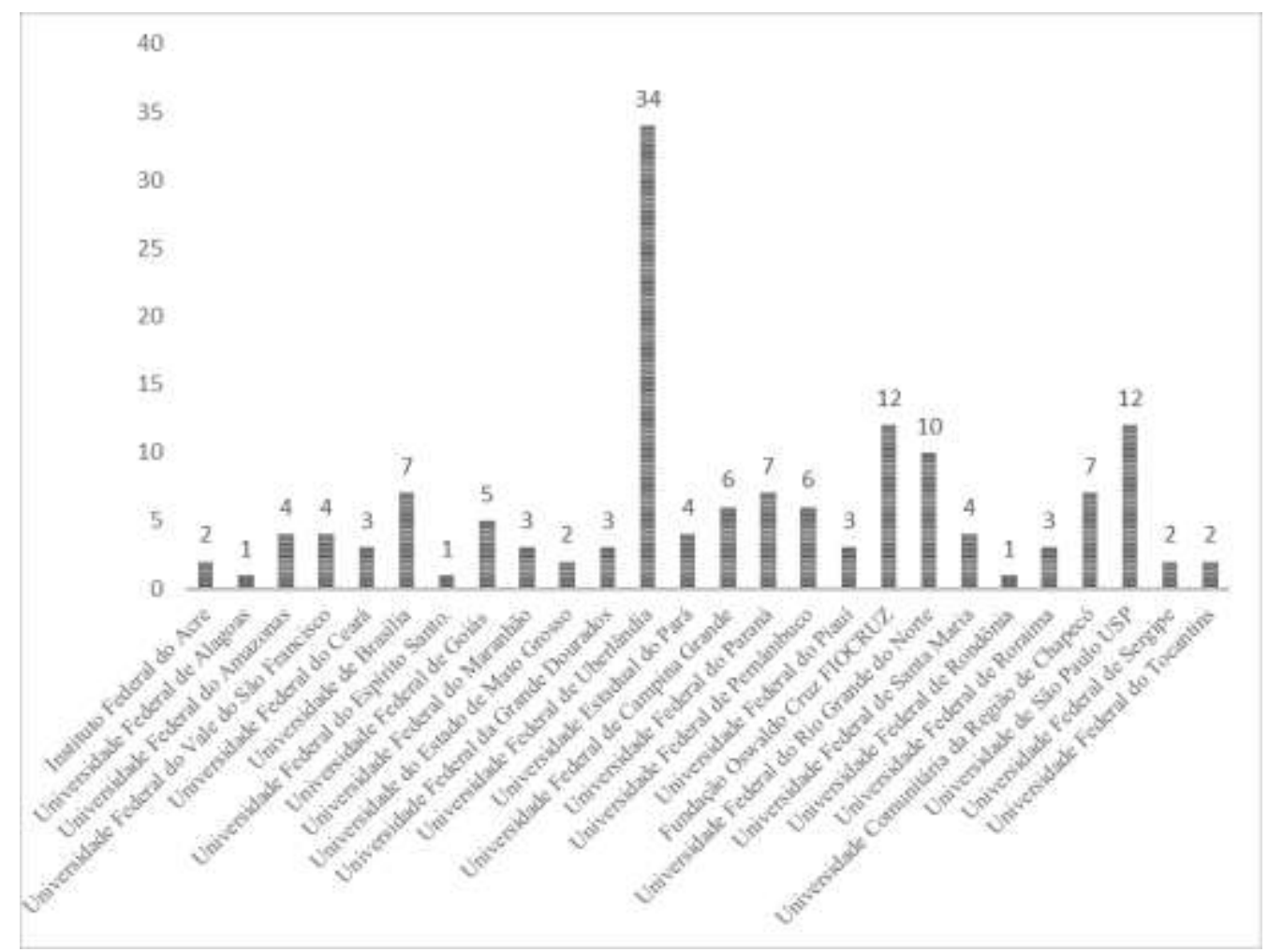

Figura 04. Levantamento das principais instituições dos autores dos artigos. Fonte: Elaborado pelos autores (2021).

Tratando-se agora dos autores que publicaram na revista, há um ótimo equilíbrio entre as publicações realizadas por homens e mulheres, o que revela que os estudos relacionados à área da saúde são bem distribuídos entre os sexos, demonstrado que essa área de estudos possui uma igualdade científica plausível, pois tal equilíbrio é muito raro na maioria dos cursos e disciplinas, nos quais há sempre a predominância ou de homens (engenharia civil, por exemplo) ou de mulheres (pedagogia), sendo, portanto, um dos pontos positivos da geografia da saúde.

Como nos mostra a figura 05, em 2012 e 2013, o número de mulheres que publicaram foi relativamente maior, já nos anos de 2014 e 2015 o número de homens que figuraram como autores e coautores foi maior, e, de 2016 a 2019 o número de mulheres voltou a ficar acima dos homens. Embora haja uma diferença de aproximadamente $20 \%$ em alguns anos, o mesmo não se mantém de forma efetiva durante todo o período analisado, o que demonstra que essa oscilação não é causada por fatores sexistas de discriminação de gênero ou de predominância de cientistas homens ou mulheres nessa área. Portanto, ao analisarmos de uma maneira geral, podemos concluir que há um equilíbrio nas publicações, revelando que o número de pesquisadores homens e mulheres se apresenta de maneira relativamente igual. 
AS CARACTERÍSTICAS DA GEOGRAFIA DA SAÚDE NO BRASIL: UMA ANÁLISE BIBLIOMÉTRICA DA PRODUÇÃO CIENTÍFICA NO PERÍODO DE 2012-2019

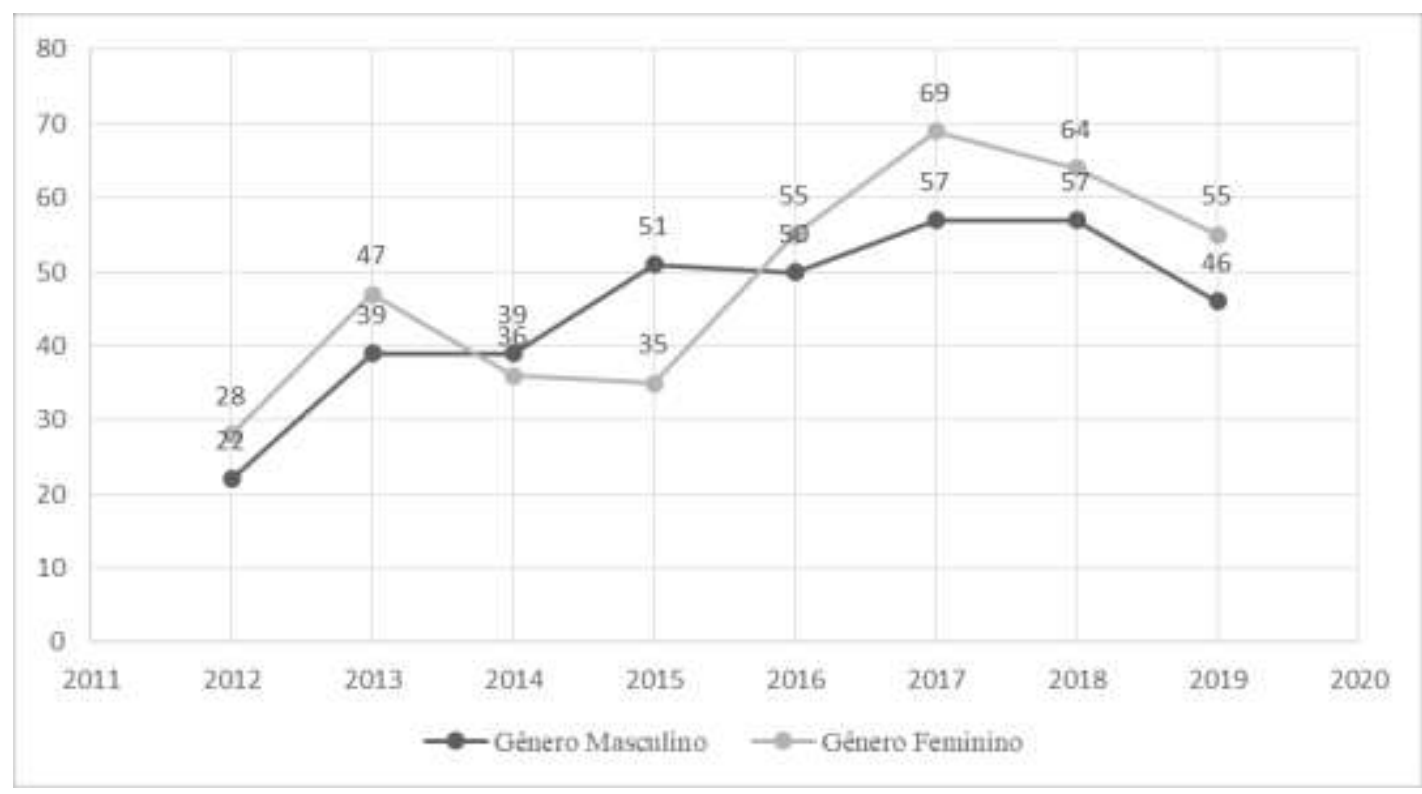

Figura 05. Relação de autores por gênero. Fonte: Elaborado pelos autores (2021).

Realizamos ainda uma análise da titulação dos autores e, como demonstrado na figura 06, a maior parte dos que publicaram na revista durante o período investigado são doutores (360), seguidos de mestres (120), e, graduandos (87). Tais dados nos revelam que a revista possui um perfil mais voltado para o de pesquisadores qualificados.

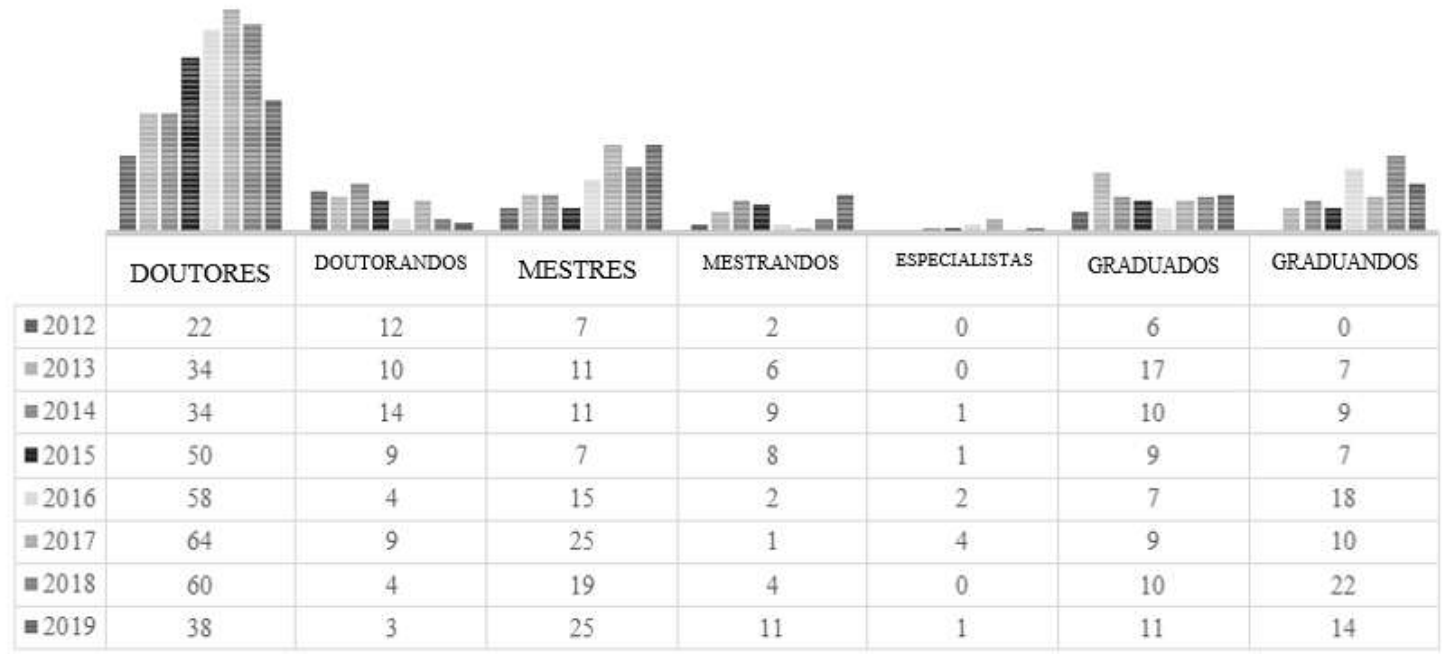

Figura 06. Titulação dos autores. Fonte: Elaborado pelos autores (2021).

Em relação aos artigos publicados na revista durante esse período, foi possível identificar as principais vertentes da Geografia da Saúde, a Nosogeográfia e a Geografia da atenção Médica. Recordando que para Peiter (2005), a primeira é considerada como um estilo mais clássico, que busca reconhecer e estudar os indicadores de distribuição espacial das doenças, ou seja, que analisa as doenças 
AS CARACTERÍSTICAS DA GEOGRAFIA DA SAÚDE NO BRASIL: UMA ANÁLISE BIBLIOMÉTRICA DA PRODUÇÃO CIENTÍFICA NO PERÍODO DE 2012-2019

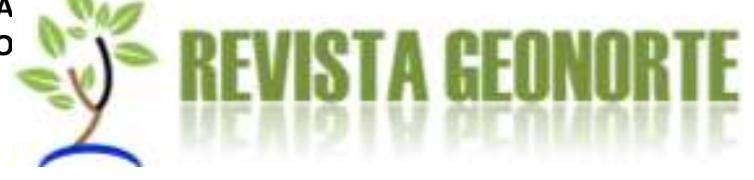

em si e suas ocorrências. Já a segunda vertente, dedica-se a conhecer como está organizado o sistema de saúde, em quais circunstâncias ele se encontra, como se dá a logística de abastecimento dos componentes infra estruturais e dos recursos humanos do sistema de atenção Médica, entre outros. Em síntese, essa vertente analisa a distribuição dos componentes dos sistemas de saúde a partir de modelos matemáticos e gráficos mais elaborados, ou seja, quantitativamente. Podemos identificar no gráfico abaixo (07), um aumento de interesse dos autores na linha da vertente da Geografia de atenção médica.

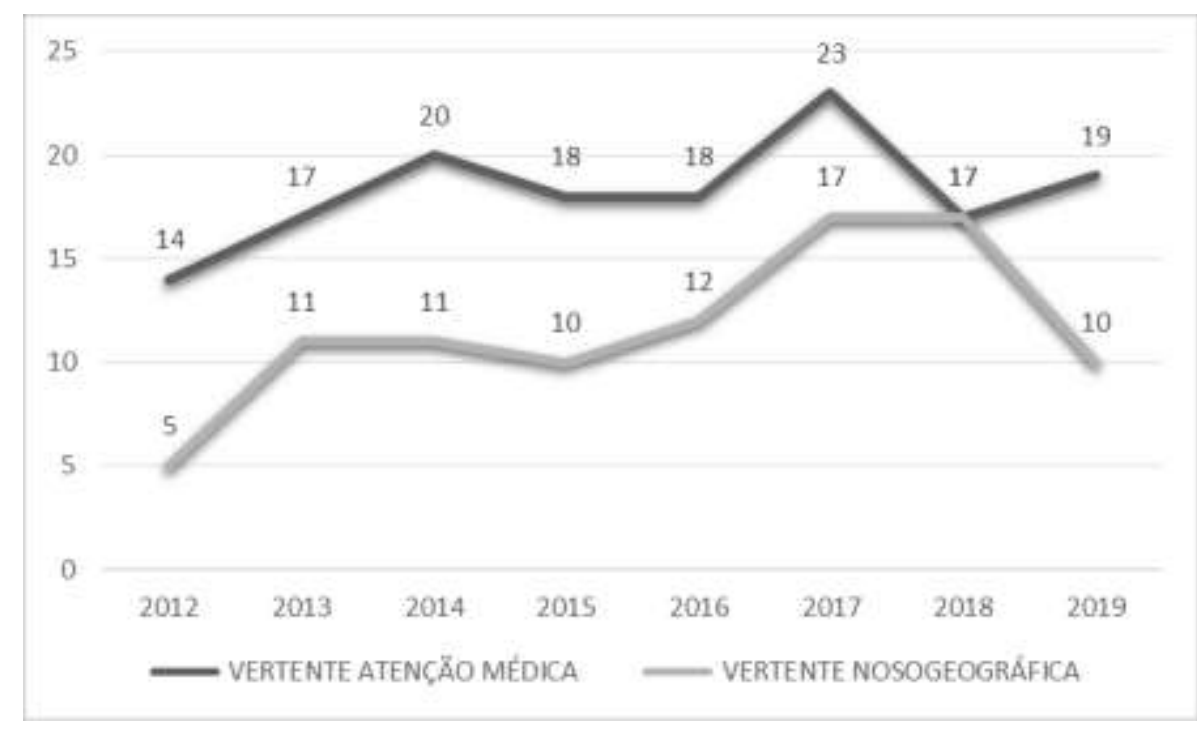

Figura 07. Vertente da Atenção Médica x Vertente Nosogeográfica. Fonte: Elaborado pelos autores (2021).

Em relação às abordagens, foi possível identificar nos artigos pesquisados a utilização dos dois principais modelos de abordagens: a abordagem ecológica e a abordagem de análise espacial. Elas têm como objetivo principal compreender o processo de saúde/doença, como estão distribuídos no espaço. Para Peiter (2005) a abordagem ecológica, caracteriza-se na investigação de enfermidades e sua relação com o meio natural, físico e relações sociais, enquanto a abordagem de análise espacial procura entender o modo como os serviços de saúde estão distribuídos e como esse arranjo influencia na qualidade de vida das diversas comunidades. Como podemos verificar no gráfico 08, houve uma tendência por parte dos autores por aderirem à abordagem da análise espacial. 
AS CARACTERÍSTICAS DA GEOGRAFIA DA SAÚDE NO BRASIL: UMA ANÁLISE BIBLIOMÉTRICA DA PRODUÇÃO CIENTÍFICA NO PERÍODO DE 2012-2019

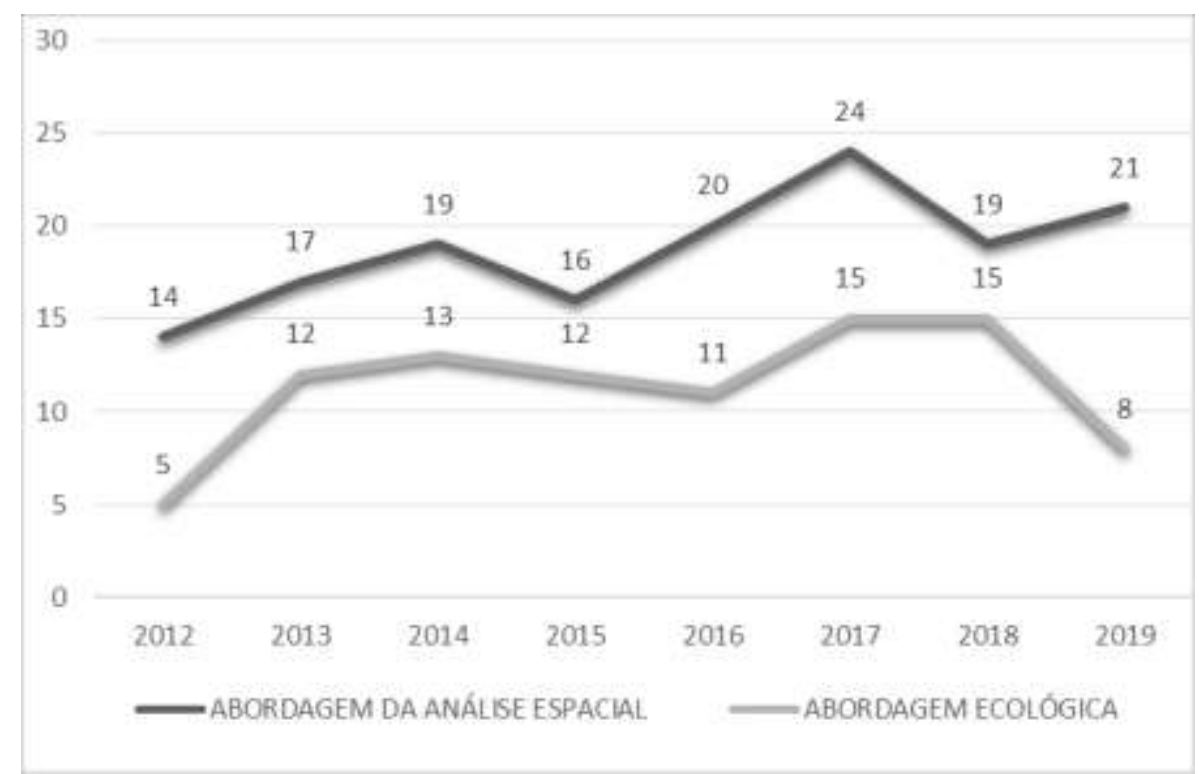

Figura 08. Abordagem da análise espacial $x$ abordagem ecológica. Fonte: Elaborado pelos autores (2021).

Quando cruzamos as informações das vertentes com as abordagens percebemos que, na maior parte das vezes em que se utilizou a Vertente da Atenção Médica para analisar, não a doença em si, mas as condições médicas de atendimento de determinada localidade, a abordagem utilizada foi a Análise Espacial e quando a vertente utilizada é a Nosogeográfica, para analisar as doenças em si e suas ocorrências, utilizou-se, quase sempre, a abordagem Ecológica, que investiga tais enfermidades a partir do meio natural, físico e cultural. Ressaltamos que não é regra que uma vertente tenha sempre uma abordagem específica.

Para uma melhor compreensão da relação entre a vertente e a abordagem em Geografia da Saúde sugere-se a leitura do trabalho de Siqueira e Martins (2019) que teve como vertente a Nosogeografia e a Abordagem Ecológica. No referido trabalho, os autores buscaram analisar a distribuição espacial da dengue na Região Administrativa de Planaltina, para o ano de 2014, focando na existência de correlação entre casos de dengue e fatores socioambientais.

Entre as doenças pesquisadas nos artigos da revista Hygeia durante o período de 2012 a 2019, a que mais apareceu foi a Dengue, seguida pela Leishmaniose Visceral e pela Hanseníase, conforme se observa na nuvem de palavras abaixo (Figura 08), na qual a quantidade de vezes que a doença foi pesquisa está relacionada proporcionalmente ao tamanho nome na figura. 
AS CARACTERÍSTICAS DA GEOGRAFIA DA SAÚDE NO BRASIL: UMA ANÁLISE BIBLIOMÉTRICA DA PRODUÇÃO CIENTÍFICA NO PERÍODO DE 2012-2019

\section{REVISTA GEONORTE}

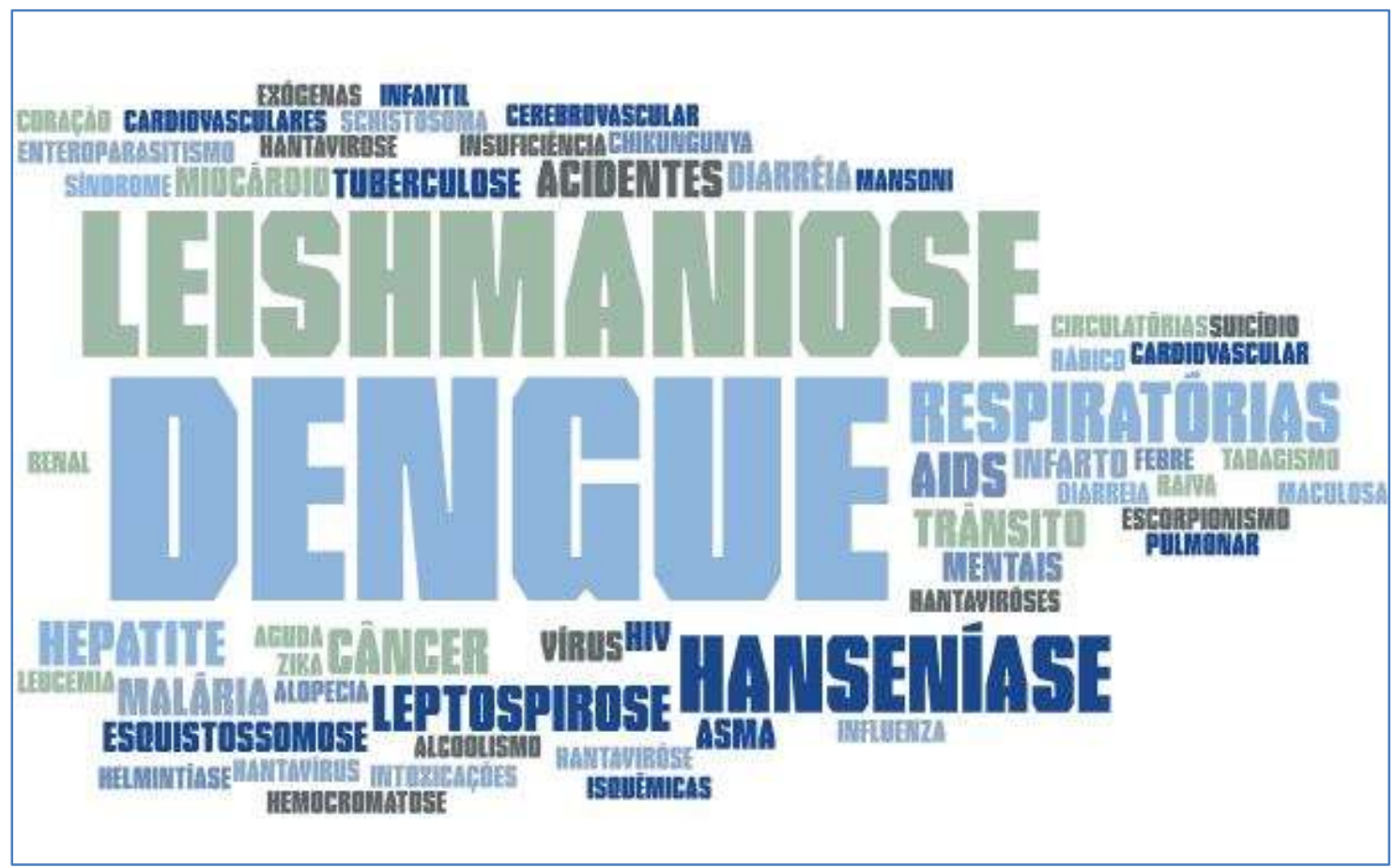

Figura 09. Nuvem de palavras sobre as principais doenças investigadas nos artigos da revista Hygeia. Fonte: Elaborado pelos autores (2021).

Algumas das doenças apresentadas acima são bastante comuns no Brasil. A Dengue e a Leishmaniose visceral, por exemplo, são propagadas através da picada de mosquitos que encontram características propícias para se propagar em nosso país, pois nossas características tipicamente tropicais e o descaso das autoridades com a prevenção propiciam isso. Atrelado a esse fator, temos também a falta de conscientização por parte da própria população, pois como sabemos, os mosquitos transmissores dessas doenças encontram terreno fértil para sua reprodução em objetos vazios que acumulam água, permitindo assim que suas lavas fecundem de maneira rápida e intensa. Já a Hanseníase é uma doença global, e que possui um histórico muito antigo no Brasil. Tais fatores parece corroborar o fato de essas doenças serem as mais recorrentes para estudos e investigações da referida revista.

Em vista de tudo, os resultados e discussões elencados acima nos mostraram as características da Geografia Médica e da Saúde no Brasil. Apesar da utilização de apenas um periódico na referida pesquisa, entretanto, considera-se que o mesmo é um dos espaços mais propícios para a área Geografia da Saúde e, que, portanto, suas publicações são retratos fieis das pesquisas e pesquisadores que se enveredaram por esta especialização da geografia.

\section{CONSIDERAÇÕES FINAIS}

A geografia da saúde se constitui como uma área de estudo científico que consegue realizar a junção das áreas humana e física da geografia. Objetiva principalmente 
AS CARACTERÍSTICAS DA GEOGRAFIA DA SAÚDE NO BRASIL: UMA ANÁLISE BIBLIOMÉTRICA DA PRODUÇÃO CIENTÍFICA NO PERÍODO DE 2012-2019

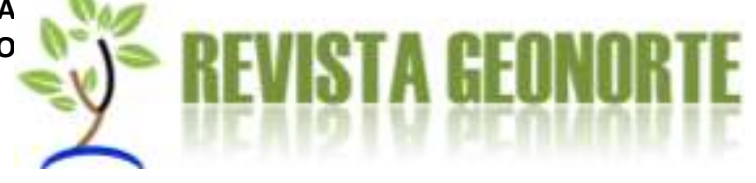

fornecer conhecimentos que nos possibilitem compreender a forma como as condições de saúde e as políticas populacionais se relacionam.

Para realizar análises geográficas relacionados à saúde, surge inicialmente a geografia médica, associada ao estudo das doenças e à materialização de mapas de difusão. Teve seu primeiro contributo no tratado da escola de Hipócrates Os ares, as águas e os lugares, e viveu seu apogeu durante o século XIX. Apesar de sua importância, não aborda o contexto no qual as doenças estão inseridas.

O século XXI acaba trazendo um novo contexto, tendo como suas principais características a concentração de população urbana e a industrialização, que acabam propiciando uma série de transformações nunca antes vista nos campos político, econômico e social. Tais condicionantes acabam revelando a má qualidade do sistema de saúde do nosso país, seguidos de medicamentos e tratamentos médicos cada vez mais caros.

Faz necessário realizar outras análises mais voltados ao contexto das doenças e os elementos propícios para tais estudos. Trabalhos nessa linha começam a surgir timidamente ainda no século XX, mas é a partir da década de 1980 que as investigações em torno da localização e do uso dos serviços de saúde começa a ganhar mais força, e é a partir de meados da década de 1990 que questões como bem estar, multiplicidade de padrões de doenças e mortes que poderiam ser evitadas começam a ser cada vez mais explorados. Tais estudos acabam fomentando a geografia da saúde.

Para entender melhor esse cenário atual, nos propomos a realizar um levantamento bibliométrico em uma das principais revistas de geografia da saúde do país. Tal metodologia é um excelente meio para identificar tendências e crescimentos do conhecimento em qualquer área de análise, sendo possível verificar o afastamento e a redução gradativa de alguns campos científicos. Além de identificar autores e instituições mais produtivos, pudemos concluir que há um aumento de interesse pela abordagem de análise espacial. Em síntese, têm-se as abordagens que deixam de lado o fenômeno em si, no caso, a doença, e enfatizam modelos matemáticos e gráficos bem elaborados, buscando analisar a distribuição dos componentes do sistema de saúde. Por outro lado, fica evidente a existência de uma estreita relação entre a abordagem ecológica com a vertente nosogeográfica, que evidencia os fenômenos através da análise de elementos ecológicos e investiga a ocorrência de doenças com tais características físicas.

Com base nesse levantamento foi possível identificar ainda a região que mais realiza publicações na revista, no caso, a região Sudeste, com $49 \%$ das publicações existentes nesse período em comparação com as outras regiões. Isso se deve ao fato de os estados que mais publicam são pertencentes à região, Minas Gerais com 66 artigos publicados, São Paulo com 32 artigos publicados e Rio de Janeiro com 24 artigos publicados. Esses dados mostram que o estudo da Geografia da Saúde apresenta um maior desenvolvimento na região Sudeste, e isso se deve à: $1^{\circ}$ ) maior quantidade de instituições de ensino com a presença de cursos voltados para a Geografia da Saúde; $2^{\circ}$ ) por serem esses cursos os mais antigos do país, fazendo 
AS CARACTERÍSTICAS DA GEOGRAFIA DA SAÚDE NO BRASIL: UMA ANÁLISE BIBLIOMÉTRICA DA PRODUÇÃO CIENTÍFICA NO PERÍODO DE 2012-2019

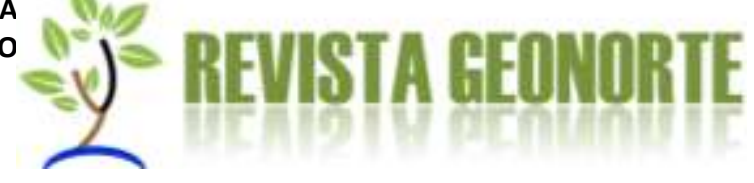

com que a gama de trabalhos produzidos nessa área possua uma demanda muito maior e mais amadurecida. Dentre as instituições de nível superior, as que lideram o ranking com o maior número de publicações são: a Universidade Federal de Uberlândia- MG (34 publicações), seguida pela Universidade de São Paulo-SP (12 publicações) e o Instituto Oswaldo Cruz-RJ (12 publicações).

Com referência ao número de homens e mulheres que publicaram na revista averiguou-se uma aproximação, na qual os homens aparecem com autores e coautores 327 vezes enquanto as mulheres aparecem 391 vezes entre autoras e coautoras. Verificando um equilíbrio de gêneros, o que não ocorre em outras áreas do conhecimento como por exemplos em áreas mais físicas. Em relação a titulação, a pesquisa mostra, que praticamente a metade, no decorrer do período analisado, dispõe de doutorado com o número de 347 doutores.

Finalmente, foi a vez de verificar os estudos das doenças mais recorrentes nas publicações e se constatou que a dengue, a Leishmaniose visceral e a hanseníase são as mais recorrentes. Isso se deve ao fato de tais doenças possuírem um terreno fértil em nosso país e da falta de conscientização por parte da população. Nesse ponto, gostaríamos de chamar a atenção para a ausência do ensino da Geografia da Saúde no Ensino Básico de nosso país. Se tal área estivesse inserida na Geografia ensinada nas escolas, sem dúvida teríamos uma maior conscientização por parte dos alunos e, consequentemente, dos pais, de modo que os altos números dessa doença poderiam ser menores

Portanto, conclui-se que os indicadores bibliométricos analisados nesse trabalho nos proporcionaram uma visão a respeito do que está se estudando no campo da Geografia da Saúde atualmente em nosso país, e tal conhecimento é importante para que possamos identificar principalmente as doenças mais estudadas e as medidas tomadas para tentar tratar das mesmas de forma coerente. Assim, a Geografia da Saúde é uma grande auxiliar para que atitudes mais assertivas sejam tomadas tanto pelos governantes quanto pela própria população.

\section{REFERÊNCIAS}

ARAÚJO, R. F.; ALVARENGA, L. A bibliometria na pesquisa científica da pósgraduação brasileira de 1987 a 2007 . Encontros Bibli: revista eletrônica de biblioteconomia e ciência da informação, 16(31), 51- 70, 2001.

ARMSTRONG, R.W. Medical Geography. In: RUFINI, J.L. (ed.). Advances in Medical Social Science. New York: Gordon and Breach Science Publishers, n.1, 1983, p. 167-183.

LAKATOS, Eva Maria. Fundamentos de metodologia científica. São Paulo: Atlas 2010. 
AS CARACTERÍSTICAS DA GEOGRAFIA DA SAÚDE NO BRASIL: UMA ANÁLISE BIBLIOMÉTRICA DA PRODUÇÃO CIENTÍFICA NO PERÍODO DE 2012-2019

MARANHÃO, Romero de Albuquerque. Análise da produção científica em geografia médica e da saúde: algumas reflexões. Caminhos de Geografia. V. 15, n. 49, Uberlândia, Mar/2014, p. 41-49.

NOSSA, P.N.S. Geografia da Saúde: o Caso da Sida. Oeiras: Celta Editora, 2001. PEITER, Paulo Cesar. A Geografia da Saúde na Faixa de Fronteira Continental do Brasil na Passagem do Milênio. 2005. 334 f. Tese (Doutorado em Geografia) Instituto de Geociências, Programa de Pós-Graduação em Geografia, Universidade Federa do Rio de Janeiro (RJ). Orientadora: Dra. Lia Osório Machado.

SANTANA, Paula. Introdução à geografia da saúde: território, saúde e bem-estar. 1 ed. Coimbra: Imprensa da Universidade de Coimbra, 2014.

Geografias da Saúde e do Desenvolvimento: evolução e tendências em Portugal. 1 ed. Almedina: Coimbra, 2005.

SANTOS, Flávia de Oliveira. Geografia médica ou Geografia da saúde? Uma reflexão. Caderno Prudentino de Geografia. V. 01, n. 32, Presidente Prudente, jan./jun. 2010.

SIQUEIRA, Rogerio Vidal de; MARTINS, Patrick Thomaz de Aquino. Casos de dengue em Planaltina-DF: análise espacial e fatores socioambientais em um ano epidêmico. Caderno de Geografia, v.29, n.58, 2019.

SNOW, J. Sobre a Maneira de Transmissão do Cólera. São Paulo/Rio de Janeiro: Hucitec/Abrasco, 1990 [1854].

SPINAK, Ernesto. Diccionário enciclopédico de bibliometría, cienciometría e informetría. Montevidéu: Unesco, 1996. 